\title{
An estimation approach for autotuning of event-based PI control systems
}

\author{
José Sánchez Moreno, María Guinaldo Losada, Sebastián Dormido \\ Departamento de Informática y Automática, UNED, C/ Juan del Rosal 16, 28040 Madrid \\ \{jsanchez, mguinaldo, sdormido\}@dia.uned.es \\ Antonio Visioli \\ Department of Mechanical and Industrial Engineering, University of Brescia, via Branze 38, 25123 \\ Brescia, Italy \{Antonio.visioli@unibs.it\}
}

\begin{abstract}
An identification procedure designed to be part of an autotuning method for event-based proportionalintegral (PI) control systems is proposed in this contribution. The rationale of the identification method is based on the information obtained from the limit cycle that the event based sampler plus an adequate tuning of the PI controller can generate in the closed loop. From the information of two limit cycles at different frequencies, the parameters of the common transfer function used for tuning of PI controllers will be deduced. Simulations demonstrate the effectiveness of the method.
\end{abstract}

Keywords: send-on-delta, limit cycle, events, identification, autotuning, PI controller.

\section{Introduction}

Methods for the identification of transfer functions parameters in event-based PI control loops have been proposed in the last years in several publications. The first investigation was described in [4]. In that work, the process parameters are estimated considering a limit cycle generated by a pre-tuned event-based PI controller. Other two methods are described in $[5,6]$. In such contributions, the rationale of the estimation methods is based on curving fitting and state-space approaches. Contributions on specific methods for identification in an event-based control loop have been recently reported in $[10,11]$; both methods are based on forcing a limit cycle.

The identification approach described in this paper is based on [11] but taking into account the full PI controller. In [11], the integral part of the controller is deactivated during the identification and only the proportional part is used to generate the limit cycle; it is also necessary to add a bias to the sampler output to introduce asymmetry in the limit cycle to calculate the dc gain. However, one of the cons described in [11] is that in lower frequencies the identification of processes with integration can be not very accurate as some of the critical points necessary to estimate the parameters are located in the first and second quadrants of the Nyquist plot (it is due to the fact that such points correspond to the third and fifth harmonics of the output system). In the procedure described here, the PI controller works on-line during the identification as the proportional and integral parts are taken into account to generate the limit cycle. Also, the issue of providing accurate results at low frequencies is worked out by adding an additional delay in order to reduce the frequency of the limit cycle.

The paper is organised as follows. In Section 2 the event-based architecture is presented. The eventbased identification procedure is described in Section 3. Section 4 explains how to improve the procedure. Finally, conclusions are given in Section 5.

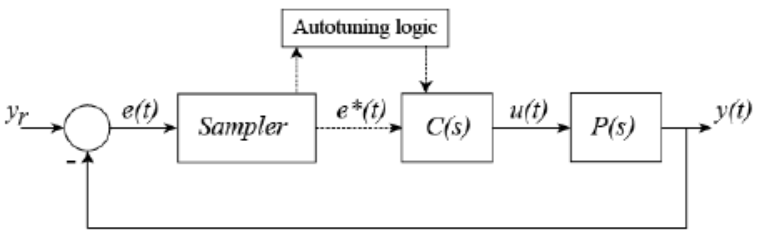

Fig. 1. Event-based control architecture

\section{Event-based PI control architecture}

The control architecture considered in this contribution is shown in Figure 1. In this event-based control system, when the sampler detects an event, it sends the information to the PI controller $C(s)$. Many logical conditions have been proposed in the literature for the occurrence of an event. The one employed here is the Symmetric Send-On-Delta (SSOD) sampling [4]. Its behaviour is described as

$$
\begin{aligned}
& e^{*}(t)= \\
& \begin{cases}(i+1) \delta & \text { if } e(t) \geq(i+1) \delta \wedge e^{*}\left(t^{-}\right)=i \delta \\
i \delta & \text { if } e(t) \in[(i-1) \delta,(i+1) \delta] \wedge e^{*}\left(t^{-}\right)=i \delta \\
(i-1) \delta & \text { if } e(t) \leq(i-1) \delta \wedge e^{*}\left(t^{-}\right)=i \delta\end{cases}
\end{aligned}
$$

With this logical condition, the sampler receives a continuous signal $e(t)$ and generates a sampled signal $e^{*}(t)$ that is multiple of $\delta$. The key of the relationship between $e(t)$ and $e^{*}(t)$ is that it can be considered as a 
generalization of a relay with hysteresis. This implies that its describing function can be derived [7].

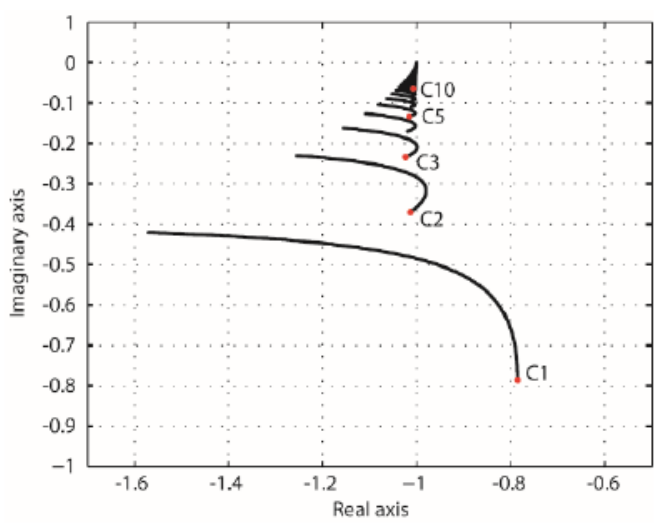

Figure 2: Nyquist plot of $-1 / N(A, \delta)$.

The describing function of the SSOD sampler is given by [9]

$$
\begin{aligned}
& N(A, \delta)= \\
& \frac{2 \delta}{\pi A}\left[1+\sqrt{1-\left(\frac{\delta}{A} m\right)^{2}}+2 \sum_{k=1}^{m-1} \sqrt{1-\left(\frac{\delta}{A} k\right)^{2}}\right] \\
& -j \frac{2 \delta^{2}}{\pi A^{2}} m
\end{aligned}
$$

where $A$ is the amplitude of a sinusoidal input signal, and $m=\lfloor A / \delta\rfloor$. The portrait of $-1 / N(A, \delta)$ is shown in Figure 2 for $A \in[\delta, \infty)$. Each intersection in Figure 2 of the system $G(s)=C(s) P(s)$ with an arc of $-1 / N(A, \delta)$ produces an oscillation (or limit cycle) of a different amplitude: Intersections with the arc starting in $C_{1}$ produce oscillations with $A \in[\delta, 2 \delta)$, intersections with the arc starting in $C_{2}$ generate oscillations with $A \in[2 \delta, 3 \delta)$, and so on. So, for example, the intersection of a system $G(s)$ with the point $C_{1}=-\frac{\pi}{4}-\frac{\pi}{4} j$ in the Nyquist map represents the existence of a limit cycle of amplitude $A=\delta$ and frequency $\omega_{\text {osc }}$; this frequency satisfies the expression $G\left(j \omega_{\text {osc }}\right)=C_{1}$.

\section{Identification procedure}

The identification method is based on the stable oscillations induced in the system $G(s)$ thanks to the existence of the event-based sampler. It must be noticed that the current process to identify must intersect the negative real axis (if not, it should be added a certain delay). Once the system is in a stable limit cycle, experimental measurements derived from the oscillatory signals are taken and used to obtain the parameters of the transfer functions used for tuning a PI controller.

The rationale of the procedure consists in forcing the system to oscillate at a frequency $\omega_{\text {osc }}$ by the detuning of the PI controller $C(s)$. As said before, the system will oscillate at $\omega_{o s c}$ as consequence of the intersection of $G(s)=C(s) P(s)$ with the reciprocal of the SSOD sampler describing function in the Nyquist map, that is,

$$
G\left(j \omega_{o s c}\right)=-\frac{1}{N(A, \delta)}
$$

As the condition for the existence of limit cycles is given by (3), a convenient detuning of $C(s)$ will produce an oscillatory behaviour of the system. So, if the Nyquist point where the system is oscillating at $\omega_{\text {osc }}$ is measured experimentally, that is, $G\left(j \omega_{o s c}\right)$, it is feasible to derive the parameters of a given transfer function model $\hat{P}(s)$.

Thus, once the system is oscillating, the procedure for fitting a model is:

(a) To measure $G\left(j \omega_{o s c}\right)$,

(b) To get the experimental value of the process at the oscillation frequency, that is, $P\left(j \omega_{o s c}\right)$, by removing $C\left(j \omega_{o s c}\right)$ from (3),

(c) To obtain $\left|P\left(j \omega_{o s c}\right)\right|$ and $\arg P\left(j \omega_{o s c}\right)$,

(d) To equate the two values obtained in the previous step to the magnitude and argument expressions of the transfer function selected to fit, and

(e) To solve the equations system and get the model parameters.

These steps are now explained in a more detailed way.

The solution adopted to get $G\left(j \omega_{o s c}\right)$ during a test is first presented in [14] and is proved in [11]. As in a limit cycle, $y(t)$ and $u(t)$ are periodic and piecewise signals, using the Laplace transform of both, it can be written

$$
G\left(j \omega_{o s c}\right)=\frac{Y\left(j \omega_{o s c}\right)}{U\left(j \omega_{o s c}\right)}=\frac{\int_{0}^{2 \pi / \omega_{o s c}} y(t) e^{-j \omega_{o s c} t} d t}{\int_{0}^{2 \pi / \omega_{o s c}} u(t) e^{-j \omega_{o s c} t} d t},
$$

where $y(t)$ and $u(t)$ are measured during a test. It must be noticed that (4) cannot be applied to determine the steady gain $\left(\omega_{\text {osc }}=0\right)$ because the oscillations produced by the SSOD block are symmetric and the integration of the periods will be zero. How the procedure is applied to get the steady gain will be 
explained afterwards, but we anticipate that the inclusion of an additional delay will play a key role.

As the PI control parameters and $\omega_{\text {osc }}$ are known, it is possible to obtain the value of $C\left(j \omega_{o s c}\right)$,

$$
C\left(j \omega_{o s c}\right)=K_{p}\left(1+\frac{1}{j \omega_{o s c} T_{i}}\right)
$$

Using (4) and (5), it is easy to obtain the experimental value of the process at the oscillation frequency,

$$
P\left(j \omega_{o s c}\right)=\frac{G\left(j \omega_{o s c}\right)}{C\left(j \omega_{o s c}\right)}
$$

The transfer function models considered in this work to explain the procedure are:

Model FOPTD:

$$
\hat{P}(s)=\frac{K}{T s+1} e^{-L s}
$$

Model IFOPTD:

$$
\hat{P}(s)=\frac{K}{s(T s+1)} e^{-L s}
$$

Model SOPTD:

$$
\hat{P}(s)=\frac{K}{(T s+1)^{2}} e^{-L s}
$$

and their argument and magnitude expressions are:

Model FOPTD:

$$
\begin{gathered}
\left|\hat{P}\left(j \omega_{o s c}\right)\right|=\frac{K}{\sqrt{T^{2} \omega_{o s c}^{2}+1}} \\
\arg \hat{P}\left(j \omega_{o s c}\right)=-\arctan \left(T \omega_{o s c}\right)-\omega_{o s c} L
\end{gathered}
$$

Model IFOPTD:

$$
\begin{gathered}
\left|\hat{P}\left(j \omega_{o s c}\right)\right|=\frac{K}{\omega_{o s c} \sqrt{1+\omega_{o s c}^{2} T^{2}}} \\
\arg \hat{P}\left(j \omega_{o s c}\right)=-\omega_{o s c} L-\arctan \left(T \omega_{o s c}\right)-\frac{\pi}{2} \\
\text { Model SOPTD: } \\
\left|\hat{P}\left(j \omega_{o s c}\right)\right|=\frac{K}{T^{2} \omega_{o s c}^{2}+1} \\
\arg \hat{P}\left(j \omega_{o s c}\right)=-2 \arctan \left(T \omega_{o s c}\right)-\omega_{o s c} L
\end{gathered}
$$

To get $K$ and $T$ it is necessary to equate $\left|P\left(j \omega_{o s c}\right)\right|$ with the magnitude of a transfer function $\left|\hat{P}\left(j \omega_{o s c}\right)\right|$ and solve the system of equations. As there are two unknowns, $K$ and $T$, in the magnitude expressions, it is necessary to run two tests to get two experimental values, that is, $P\left(j \omega_{o s c_{-} 1}\right)$ and $P\left(j \omega_{o s c_{-} 2}\right)$. Notice that each test will be run with a different set of control parameters to force the system to oscillate at different frequencies, that is, $\omega_{o s c_{-} 1}$ and $\omega_{o s c_{-} 2}$. It will be explained how to modify the PI parameters in the following paragraphs depending on the process and the model to identify. Once $K$ and $T$ are known, the delay $L$ is obtained by equating $\arg P\left(j \omega_{\text {osc_1 }}\right)$ with the argument expression of the selected transfer function model to fit, that is, with (11), (13) or (15).

The following expressions are the result of solving the equations for the three models. For the sake of simplicity, $\omega_{i}$ represents $\omega_{\text {osc_i }},\left|P_{i}\right|$ represents $\left|P\left(\omega_{\text {osc }_{-} i}\right)\right|, \quad$ and $\arg P_{i}$ corresponds to $\arg P\left(j \omega_{o s c_{-} i}\right)$.

\section{Model FOPTD:}

$$
\begin{gathered}
K=\sqrt{\frac{\omega_{1}^{2}-\omega_{2}^{2}}{\left|P_{1}\right|^{2} \omega_{1}^{2}-\left|P_{2}\right|^{2} \omega_{2}^{2}} \mid P_{1}}|| P_{2} \mid \\
T=\sqrt{\frac{\left|P_{2}\right|^{2}-\left|P_{1}\right|^{2}}{\left|P_{1}\right|^{2} \omega_{1}^{2}-\left|P_{2}\right|^{2} \omega_{2}^{2}}} \\
L=-\frac{\arg P_{1}+\arctan \left(T \omega_{1}\right)}{\omega_{1}}
\end{gathered}
$$

Model FOPTDI:

$$
\begin{gathered}
K=\sqrt{\frac{\omega_{1}^{2}-\omega_{2}^{2}}{\left|P_{1}\right|^{2} \omega_{1}^{4}-\left|P_{2}\right|^{2} \omega_{2}^{4}}}\left|P_{1}\right|\left|P_{2}\right| \omega_{1} \omega_{2} \\
T=\sqrt{\frac{\left|P_{2}\right|^{2} \omega_{2}^{2}-\left|P_{1}\right|^{2} \omega_{1}^{2}}{\left|P_{1}\right|^{2} \omega_{1}^{4}-\left|P_{2}\right|^{2} \omega_{2}^{4}}} \\
L=-\frac{\arg P_{1}+\arctan \left(T \omega_{1}\right)+0.5 \pi}{\omega_{1}}
\end{gathered}
$$

Model SOPTD:

$$
\begin{gathered}
K=\frac{\omega_{1}^{2}-\omega_{2}^{2}}{\left|P_{1}\right| \omega_{1}^{2}-\left|P_{2}\right| \omega_{2}^{2}\left|P_{1}\right|\left|P_{2}\right|} \\
T=\sqrt{\frac{\left|P_{2}\right|-\left|P_{1}\right|}{\left|P_{1}\right| \omega_{1}^{2}-\left|P_{2}\right| \omega_{2}^{2}}} \\
L=-\frac{\arg P_{1}+2 \arctan \left(T \omega_{1}\right)}{\omega_{1}}
\end{gathered}
$$

As said before, it is necessary to run two tests to measure $G\left(j \omega_{o s c_{-} 1}\right)$ and $G\left(j \omega_{o s c_{-} 2}\right)$. The first test is done just by increasing the proportional gain $K_{p}$ until the system reaches a limit cycle and oscillates at a frequency $\omega_{o s c} 1$. The second test is prepared by a 
second increase of $K_{p}$ to reach a new limit cycle at another frequency $\omega_{o s c_{-} 2}>\omega_{\text {osc }_{-} 1}$.

However, the previously defined procedure just works when the current process and the model to fit have the same order and structure. This is due to the following reasons:

- If the transfer function template to fit $\hat{P}(s)$ is exactly equal to the actual process to identify, the identification procedure will provide an exact result. This is due to the fact that the template is fitted with the same degrees of freedom than the true process. As result, the behaviour of $\hat{G}(s)$ will be equal to $G(s)$ in all the frequencies range.

- If the process has a higher order than the template or a different structure, this will produce the result to be exact at the range of frequencies between $\omega_{o s c_{-} 1}$ and $\omega_{o s c_{-} 2}$ but with discrepancies at other frequencies. This is a consequence of fitting the template with lesser degrees of freedom than the true process. The effect is that the behavior of the model at frequencies out of the range Modofy can become very inaccurate. Such fact will be especially notorious and visible at frequencies below $\omega_{o s c_{-} 1}$ or at the steady state when the fitted model is a FOPTD or a SOPTD, that is, when the current process does not have integral dynamics.

The solution proposed in this work consists of forcing the system to oscillate during the second test at a very low frequency as close to zero as possible. To reduce the frequency of the limit cycle below $\omega_{\text {osc_1 }}$ an additional delay will be added to the system during the second test. Next some examples are given in order to explain better the problem and present the solution.

\subsection{Identification of IFOPD processes}

Example 1: To start illustrating the event-based identification procedure, let considering the process [8],

$$
P(s)=\frac{e^{-0.2 s}}{s(s+1)}
$$

Initially, the process is controlled by a PI tuned to force the system to oscillate. The controller parameters selected for such a goal are $\left[K_{p}=1, T_{i}=10\right]$. In all the simulations, measurement noise was not considered and $\delta$ was set to 1 . The data obtained in the first test were
$G\left(j \omega_{\text {osc_1 }}\right)=(-0.8542-0.6357 j)$ at frequency $\omega_{\text {osc_1 }}=0.755$. For the second test, $K_{p}$ was increased to 1.2 to obtain a limit cycle at a higher frequency and $T_{i}$ was not changed. Now, the second test data were $\omega_{o s c_{-} 2}=0.8671$ and $G\left(j \omega_{\text {osc_2 }}\right)=(-0.8878-0.5652 j)$. The model parameters were obtained by applying (5) and (6) to the previous data to get $P\left(j \omega_{o s c_{-} 1}\right)$ and $P\left(j \omega_{o s c_{-} 2}\right)$, and after that, using (19), (20) and (21). The resulting model and results obtained from other relay-based identification methods are presented in Table I. It can be appreciated that the event-based procedure gives results of the same quality as more elaborated methods based on state-space [1] and curve-fitting [8] approaches.

Table I: Models and errors for (25) where $\omega_{p c}=2.16$.

\begin{tabular}{|c|c|c|}
\hline Method & Model & $\widetilde{E}$ \\
\hline $\begin{array}{c}\text { Event-based } \\
\text { procedure }\end{array}$ & $\frac{1.0000 e^{-0.2015 s}}{s(1.0000 s+1)}$ & 0.00349 \\
\hline By [8] & $\frac{1.0000 e^{-0.2 s}}{s(0.9998 s+1)}$ & 0.00027 \\
\hline By [1] & $\frac{1.0000 e^{-0.2 s}}{s(0.9999 s+1)}$ & 0.00013 \\
\hline
\end{tabular}

The accuracy of the estimated process model is computed using the frequency domain estimation error index $(\widetilde{E})$ for each of the process models is found by applying integral of absolute error (IAE) criterion as

$$
\widetilde{E}=\int_{0}^{\omega_{p c}}\left|\frac{P(j \omega)-\hat{P}(j \omega)}{\hat{P}(j \omega)}\right| d \omega,
$$

where $\omega_{p c}$ is the phase cross over frequency of the actual process $P(s)$, that is, the frequency where phase shift is equal to $-180^{\circ}$.

Example 2: Let now considering the identification of the higher-order process presented in [8],

$$
P(s)=\frac{(-s+1) e^{-5 s}}{s(s+1)^{5}}
$$

After two consecutive tests with the two set of control parameters $\quad\left[K_{p}=0.1, T_{i}=50\right] \quad$ and $\left[K_{p}=0.12, T_{i}=50\right]$, the model obtained is shown and compared in Table II. The obtained data were $\omega_{\text {osc_1 }}=0.098$ and $P\left(j \omega_{\text {osc_1 }_{-}}\right)=(-8.8169-4.7364 j)$ 
for the first test, and $\omega_{o s c_{-} 2}=0.1167$ and $P\left(j \omega_{\text {osc }_{-} 2}\right)=(-7.996-2.3685 j)$ for the second one.

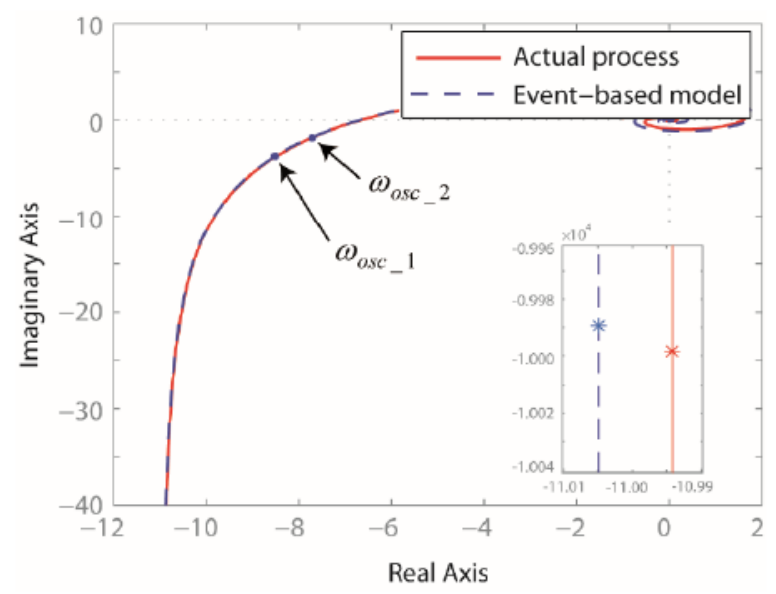

Figure 3: Plots of (26) and the identified model.

In the Example 2, the Nyquist plots of the model and the process are apparently similar in the third quadrant (see Figure 3). In particular, the model and the process behave in a similar way between $\omega_{o s c_{-}} 1$ and $\omega_{o s c_{-} 2}$. However, there are discrepancies at lower frequencies. Indeed, at the frequencies $\omega=0.01,0.001$ and 0.0001 , the differences between the true process and model, that is, $|P(j \omega)-\hat{P}(j \omega)|$, are $0.088,0.87$, and 8.73 , respectively (see detail in Figure 3 of the Nyquist points at $\omega=0.001$ ).

Table II: Models and errors for (26) where $\omega_{p c}=0.143$.

\begin{tabular}{|c|c|c|}
\hline Method & Model & $\widetilde{E}$ \\
\hline $\begin{array}{c}\text { By the event- } \\
\text { based } \\
\text { procedure }\end{array}$ & $\frac{0.9991 e^{-9.0526 s}}{s(1.9670 s+1)}$ & 0.00019 \\
\hline By [8] & $\frac{0.7319 e^{-0.5 s}}{3.1953 s(2.0051 s+1)}$ & 0.52121 \\
\hline By [13] & $\frac{1.018 e^{-8.5278} s}{s(2.5293 s+1)}$ & 0.00156 \\
\hline
\end{tabular}

\subsection{Identification of FOPTD processes}

Example 3: Consider the following process

$$
P(s)=\frac{e^{-2 s}}{10 s+1}
$$

that is being well controlled by a SSOD-PI tuned with $\left[K_{p}=2, T_{i}=10\right]$. By increasing the proportional gain, the sets of parameters used to enter the system into two different stable limit cycles are found to be $\left[K_{p}=5, T_{i}=10\right]$ and $\left[K_{p}=6, T_{i}=10\right]$. The results of the fitting can be found in Table III. It must be noticed that the result of the event-based procedure is very accurate because the actual process has the same order that the template to fit.

Example 4: Now the following high-order process is going to be identified as a FOPTD model

$$
P(s)=(s+1)^{-4}
$$

where $\omega_{p c}=1$. Applying the procedure as before, that is, with two sets of control parameters that force the system to oscillate, for example, $\left[K_{p}=1.5, T_{i}=3\right] \quad$ and $\left[K_{p}=1.6, T_{i}=3\right]$, the estimated model is

$$
\hat{P}(s)=\frac{2.9113 e^{-1.1751 s}}{8.7939 s+1}
$$

Table III: Models and errors for (27) where $\omega_{p c}=0.844$.

\begin{tabular}{|c|c|c|}
\hline Method & Model & $\widetilde{E}$ \\
\hline $\begin{array}{c}\text { By the event-based } \\
\text { procedure }\end{array}$ & $\frac{0.9999 e^{-2.0015 s}}{9.9999 s+1}$ & 0.00054 \\
\hline By [2] & $\frac{0.999 e^{-2.0 s}}{9.9957 s+1}$ & 0.00055 \\
\hline By [12] & $\frac{1.03 e^{-2.3 s}}{10.3 s+1}$ & 0.1140 \\
\hline
\end{tabular}

Obviously, such result is not acceptable as the steady gain is far from the correct value of one producing an estimation error index very high $(\widetilde{E}=0.347)$. With the identification procedure as originally defined, the fitting of the model is good around the two Nyquist points defined by the frequencies of the two limit cycles but not at $\omega \approx 0$. In this example, such oscillations frequencies are $\omega_{\text {osc_1 }_{1}}=0.5506$ and $\omega_{\text {osc_2 }}=0.5739$, and the differences are small,

$$
\begin{aligned}
\left|P\left(j \omega_{o s c_{-} 1}\right)-\hat{P}\left(j \omega_{o s c_{-} 1}\right)\right| & =0.0004 \\
\left|P\left(j \omega_{o s c_{-} 2}\right)-\hat{P}\left(j \omega_{o s c_{-} 2}\right)\right| & =0.01949
\end{aligned}
$$

but not for $\omega=0$,

$$
|P(0)-\hat{P}(0)|=1.9113
$$

It can be observed in Figure 4 that the identified model fits correctly around the oscillation frequencies measured in the two tests. In particular, the fitting is exact for $\omega_{o s c_{-} 1}$ as it is the frequency 
selected for getting $L$ with (18) once $K$ and $T$ are known by (16) and (17).

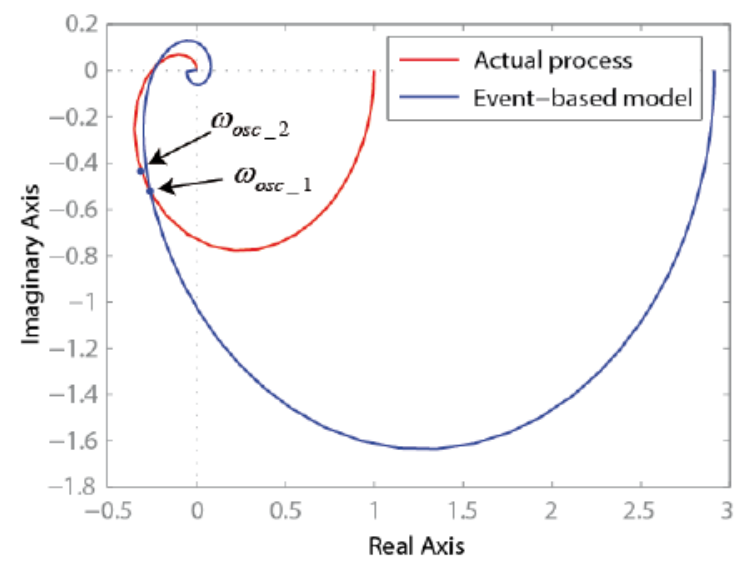

Figure 4: Nyquist plots of (28) and the fitted model.

If a fitting of a SOPTD model is tried, the new result improves with respect to the previous FOPTD model,

$$
\hat{P}(s)=\frac{1.1110 e^{-0.9142 s}}{(1.7102 s+1)^{2}}
$$

with $\widetilde{E}=0.0753$, but there is still a $10 \%$ of discrepancy at $\omega \approx 0$,

$$
|P(0)-\hat{P}(0)|=0.1110
$$

\section{$4 \quad$ Modifying the procedure}

A practical solution to make a correct identification is to generate in the second test a limit cycle at a frequency $\omega_{\text {osc } 2}$ as near zero as possible. A point of $G(s)$ with a frequency $\omega_{\text {osc } 2}<<\omega_{\text {osc_1 }}$ are, in general, far from the intersection with the DF of the even-based sampler and, also, due to the integral action of the PI controller, the point will be located along the negative real axis of the Nyquist map. The solution is to add new dynamics to $G(s)$ to allow that the very low frequency range of the new system $G^{\prime}(s)$ intersects in some point with $-1 / N(A, \delta)$. To understand how to modify the estimation procedure to make the second test with a low frequency limit cycle, see the steps depicted in Figure 5.

Step 1 consists in rotating a unknown Nyquist point $P_{1}=G\left(j \omega_{\text {osc } \_2}\right)$, where $\omega_{o s C_{-} 2}$ is a very low frequency, to the grey area depicted in Figure 5. That area represents the theoretical section of the Nyquist map where the intersection of $G^{\prime}(s)$ with the negative reciprocal of $N(A, \delta)$ can be produced after a radial movement of the point $P_{2}$ (Step 2). This theoretical section is located between $\arg (-1 / N(\infty, \delta))=-\pi \quad$ and $\arg (-1 / N(\delta, \delta))=-0.75 \pi$, that is, between $-180^{\circ}$ and $-135^{\circ}$.

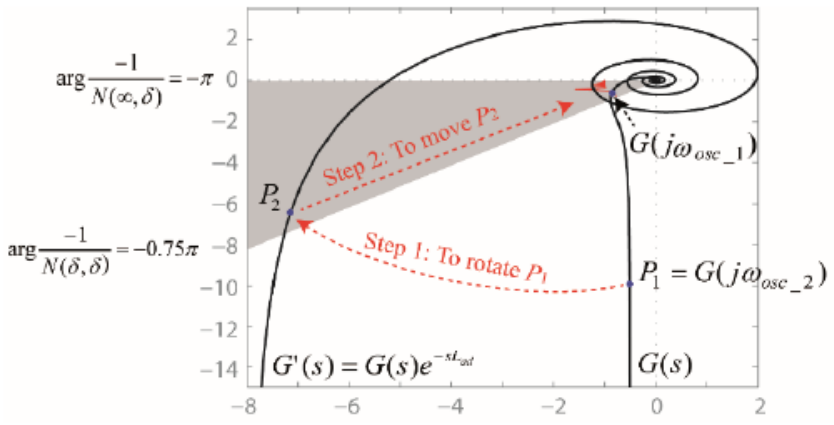

Figure 5: Steps to modify the second test to get oscillations at frequencies near zero.

A way to get that is by rolling $G(s)$ around the center of Nyquist map. As the rotation can be done by adding a delay $L_{a d}$ to $G(s)$, that is, $G^{\prime}(s)=G(s) e^{-s L_{a d}}$, bounds for $L_{a d}$ to assure that $P_{1}$ will be rotated inside the grey area are given by,

$$
\frac{-\pi-\arg P_{1}}{\omega_{\text {osc_2 }} 2}<-L_{a d}<\frac{-0.75 \pi-\arg P_{1}}{\omega_{o s c_{-} 2}}
$$

Assuming that the frequency $\omega_{o s c_{-} 2}$ selected for the second test is low enough (e.g., $0.1 \omega_{o s c_{-} 1}$ ), and because of the integral action of the PI controller, we can consider $\arg P_{1} \approx-0.5 \pi$ (that is, $-90^{\circ}$ ) at very low frequencies. Thus, from (29) practical bounds for $L_{a d}$ could be,

$$
\frac{0.5 \pi}{\omega_{o s c_{-} 2}}>L_{a d}>\frac{0.25 \pi}{\omega_{\text {osc_2 }}}
$$

and fixing $\omega_{o s c_{-} 2}=0.1 \omega_{o s c_{-} 1}$,

$$
\frac{5 \pi}{\omega_{\text {osc_1 }}}>L_{a d}>\frac{2.5 \pi}{\omega_{\text {osc_1 }}}
$$

Now, as the unknown point $P_{2}=G\left(j \omega_{\text {osc_2 } 2}\right) e^{-j \omega_{\text {osc }}{ }_{2} L_{a d}}$ is located in the grey area but far from the intersection with $-1 / N(A, \delta)$, it is necessary to give a second step. This step consists in a radial translation of the new system $G(s) e^{-s L_{a d}}$ looking for an intersection with $-1 / N(A, \delta)$. That must be done by reducing the proportional gain as it can be appreciated in Figure 5. Unfortunately, the calculation of this gain is not intuitive and must be done by trial and error. 
Example 5: We identify the previous process $P(s)=(s+1)^{-4}$ by applying the modified procedure. The first test is run with the same parameters as in the previous example, that is, $\left[K_{p}=1.5, T_{i}=3\right]$, and the result is $\omega_{\text {osc_1 }}=0.55067$ and $\arg G\left(j \omega_{\text {osc_1 } 1}\right)=-146^{\circ}$. By fixing $\omega_{o s c_{-} 2}=0.1 \omega_{o s c_{-} 1}$ and according to (30), we obtained $28.5>L_{a d}>14.2$. The second test is run with the following set of parameters $\left[K_{p}=0.15, T_{i}=3, L_{a d}=21.4\right]$ and the frequency measured is $\omega_{\text {osc_2 }}=0.049$, that is close to $0.1 \omega_{o s c_{-} 1}$. In Table IV and Figure 6, the new estimation is presented and compared with the model obtained by a more elaborated method.

Table IV: FOPTD models of (28) where $\omega_{p c}=1$.

\begin{tabular}{|c|c|c|}
\hline Method & Model & $\widetilde{E}$ \\
\hline $\begin{array}{c}\text { By the event-based } \\
\text { procedure }\end{array}$ & $\frac{1.0026 e^{-1.9450 s}}{2.5032 s+1}$ & 0.0993 \\
\hline By [3] & $\frac{0.987 e^{-1.889 s}}{3.1036 s+1}$ & 0.1426 \\
\hline
\end{tabular}

Example 6: Table $\mathrm{V}$ shows the fitting of $P(s)=(s+1)^{-4}$ to a SOPTD model using the results of the two tests of the Example 5.

As said before, if the structure of the actual process and the model to fit are the same, the original method is valid for any model and it is not necessary in the second test to force the system to oscillate at a very low frequency. But if the structure of the actual process is higher than the model template it will be necessary to modify the method as explained before.

However, the original method is valid for FOPTDI fitting of high-order processes with one pole at the origin (see Example 7). It is due to the double integral action introduced by the process and the controller. Forcing the second limit cycle at a very low frequency can be done by reducing the proportional gain used in the first test. The effect of this action produces two consequences in the Nyquist plot of $G(s)$ : (a) to be moved radially towards the origin, and (b) the reduction of the phase margin as consequence of a lower integral gain $\left(K_{p} / T_{i}\right)$. The radial movement produced an approach of the low frequencies to the origin, and the reduction of the phase margin reassures the intersection of $G(s)$ with the reciprocal of the describing function of the eventbased block.

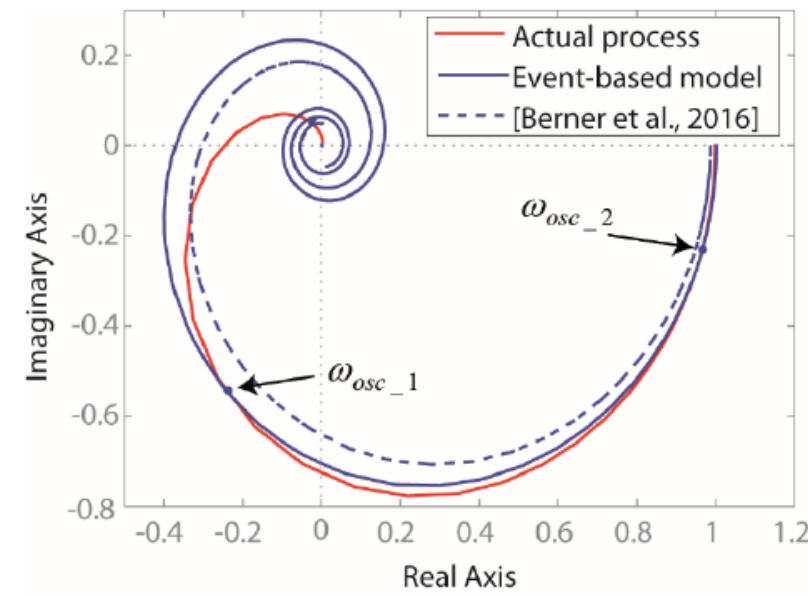

Figure 6: Nyquist plots of $P(s)=(s+1)^{-4}$ and the identified model with the modified procedure.

Table V: SOPTD models of (28) where $\omega_{p c}=1$.

\begin{tabular}{|c|c|c|}
\hline Method & Model & $\widetilde{E}$ \\
\hline $\begin{array}{c}\text { By the event-based } \\
\text { procedure }\end{array}$ & $\frac{1.0007 e^{-1.127 s}}{(1.5189 s+1)^{2}}$ & 0.0472 \\
\hline By [3] & $\frac{1.054 e^{-1.004 s}}{(1.762 s+1)^{2}}$ & 0.0827 \\
\hline
\end{tabular}

Example 7: To produce a new limit cycle at a very low frequency using the process of the Example 2, a new simulation is run with the control parameters $\left[K_{p}=0.001, T_{i}=50\right]$. It must be noticed that the proportional gain has been significantly reduced with respect to the parameters applied in the second test in Example 2 (that are $\left[K_{p}=0.12, T_{i}=50\right]$ ). Now, the frequency of this new limit cycle is $\omega_{\text {osc_2_new }}=0.004$. It can be observed in Figure 7 the differences in the frequencies of the limit cycles depending on the selected set of controller parameter. The identified FOPTDI model of (26) using data from the limit cycles at $\omega_{\text {osc_1 }}=0.098$ and $\omega_{\text {osc_2 }}=0.004$ is

$$
\hat{P}(s)=\frac{1.0001 e^{-8.9984 s}}{s(2.0233 s+1)}
$$

With this new model, the discrepancies at lower frequencies with respect to (26) have been reduced. For the frequencies $\omega=0.01,0.001$ and 0.0001 , the differences $|P(j \omega)-\hat{P}(j \omega)|$ are $0.027,0.167$, and 1.66 , respectively (compare these values with those presented at the end of Example 2). 


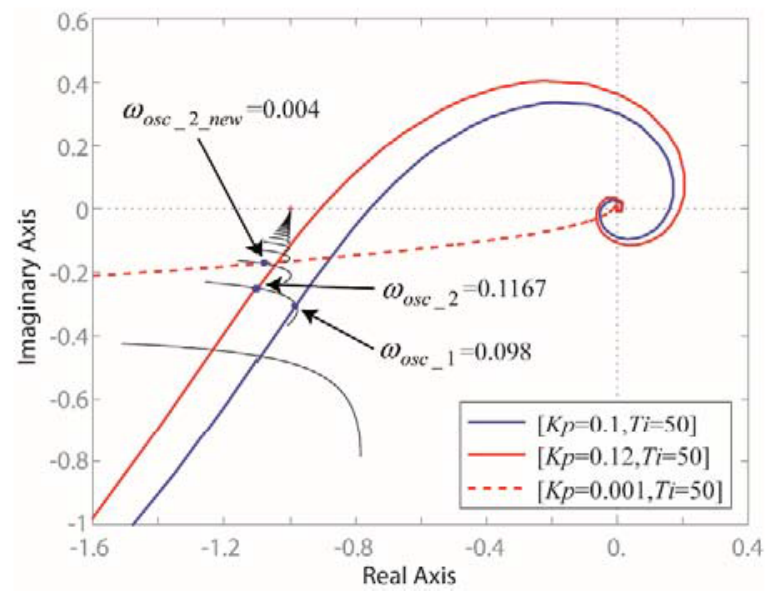

Figure 7: Plots of $G(s)$ where

$$
\begin{gathered}
P(s)=(-s+1) e^{-5 s} /(s+1)^{5} \text { and } C(s) \text { changes its } \\
\text { parameters. }
\end{gathered}
$$

\section{Conclusions}

In this paper, an autotuning method completely designed for event-based PI control loops has been presented. The identification approach is based on the information obtained from two limit cycles produced by the SSOD sampler and the PI controller. Simulation examples have proven the effectiveness of the method. However, there are some issues that need to be improved.

For example, regarding the identification of FOPTD and SOPTD models, it is necessary to improve the procedure to determine the second test, especially the estimation of the new proportional gain to apply in the Step 2. This will be part of future investigations.

\section{Acknowledgements}

Work funded by Spanish Ministry of Economy and Competitiveness under contracts DPI2014-55932C2-2-R and DPI2017-84259-C2-2-R.

\section{References}

[1] Bajarangbali, S., Mahji, J. (2015) "Identification of integrating and critically damped systems with time delay", Control Theory and Technology 13(1), pp. 29-36.

[2] Bajarangbali R., Majhi S., Pandey S. (2014) "Identification of FOPDT and SOPDT process dynamics using closed loop test", ISA Transactions 53(4), pp. 1223-1231.

[3] Berner J., Hägglund T., Åström K.J. (2016) "Asymmetric relay autotuning - Practical features for industrial use", Control Engineering Practice 54, pp. 231-245.
[4] Beschi, M., Dormido, S., Sánchez, J, Visioli, A., Yebra, L.J. (2014) "Event-based PI plus feedforward control strategies for a distributed solar collector field", IEEE Transactions on Control Systems Technology 22(4), pp. 1615-1622.

[5] Beschi, M., Dormido, S., Sánchez, J, Visioli, A. (2015) "Closed-loop automatic tuning technique for an event-based PI controller", Ind. Eng. Chem. Res. 54(24), pp. 6362-6370.

[6] Beschi, M., Dormido, S., Sánchez, J, Visioli, A. (2015) "An Event-based PI Controller Autotuning Technique for Integral Processes". Ist $^{\text {IEEE }}$ International Conference on Event-Based Control, Communication, and Signal Processing (EBCCSP2015). , Krakow (Poland).

[7] Gelb, A; Van der Velde, WW. (1968) MultipleInput Describing Functions and Nonlinear System Design. New York, USA: McGraw-Hill.

[8] Panda, C., Vijayan, V. Sujatha, V. (2011) "Parameter estimation of integrating and time delay processes using single relay feedback test", ISA Trans 50(4), pp. 529-537.

[9] Romero J.A., Sanchís, R. (2016) "A new method for tuning PI controllers with symmetric send-ondelta sampling strategy", ISA Trans 64, pp. 161-173.

[10] Sánchez, J., Guinaldo, M., Dormido, S., Visioli, A. (2018) "Identification of process transfer function parameters in event-based PI control loops", ISA Trans 75, pp. 157-171.

[11] Sánchez, J., Guinaldo, M., Dormido, S., Visioli, A. (2018) "Enhanced event based identification procedure for process control. Ind. Eng. Chem. Res. DOI: 10.1021/acs.iecr.7b05239.

[12] Srinivasan, K., Chidambaram, M. (2003) "Modified relay feedback method for improved system identification", Comput. Chem. Eng. 27(5), pp. 727-732.

[13] Tao, L., Furong, G. (2008) "Identification of integrating and unstable processes from relay feedback", Comput. Chem. Eng. 32(12), pp. 30383056.

[14] Vivek, S., Chidambaram, M. (2005) "Identification using single symmetrical relay feedback test". Computers \& Chemical Engineering 29(7), pp. 1625-1630.

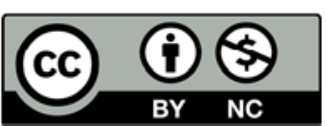
(c) 2018 by the authors. Submitted for possible open access publication under the terms and conditions of the Creative Commons Attribution CC-BY-NC 3.0 license (https://creativecommons.org/licenses/by-nc/3.0). 